\title{
Utilizing NMR to study RNA- and compound-binding mechanisms of Musashi-1, a stem/progenitor cell marker in various normal and cancer cells
}

\author{
Takashi Nagata ${ }^{1,2 *}$ \\ ${ }^{1}$ Institute of Advanced Energy, Kyoto University, Japan \\ ${ }^{2}$ Graduate School of Energy Science, Kyoto University, Japan
}

An RNA-binding protein, Musashi-1 (Msi1), is a posttranscriptional gene regulator that is involved in the regulation of stem cell self-renewal. Because of its high-level expression in neural stem cells (NSCs) and undifferentiated neural precursor cells [1], Msi1 is used as a cell marker for NSCs and progenitor cells in the central nervous system. Increasing evidence suggested that Mis1 acts as a critical regulator of the status of stem/progenitor cells in other tissues and organs [2,3], including the breast, eye, hair follicles, intestine, and stomach, in either embryonic or adult stages; thus, Msil may also be used as an effective marker for stem/progenitor cells in a wide range of tissues and organs.

The well-established function of Msi1 is repression of translation of Numb and APC, which are antagonists of Notch and Wnt signaling, respectively [4-6], and also repression of translation of cyclindependent kinase inhibitor $\mathrm{P} 21^{\mathrm{WAF}-1}$, which is a negative regulator of cell cycle progression [7]. Later, various high-throughput genome-wide analyses identified substantial number of Msi1-targeted mRNAs [2,3], each encoding a protein involved in cellular activity such as apoptosis, cell cycle regulation, differentiation, proliferation, survival, and DNA repair.

Msil has two tandemly connected ribonucleoprotein-type RNAbinding domains (RBDs), RBD1 and RBD2, in its N-terminal region. Previously, we applied NMR methods to Msi1 RBD1 and RBD2 individually, and determined the solution structures in their free forms, identified the residues involved in RNA-binding, and assessed the backbone dynamics and discussed the origin of high RNA-binding affinity $[8,9]$. Our subsequent NMR analysis identified the minimal recognition RNA sequences for Msi1 RBD1 and RBD2 to be $\mathrm{r}(\mathrm{GUAG})$ and $\mathrm{r}(\mathrm{UAG})$, respectively [10]. Then, we determined the NMR solution structure of Msi1 RBD1-r(GUAGU) complex, which clearly revealed the detailed interactions between Msil RBD1 and r(GUAG) [10]. Furthermore, a theoretical analysis based on statistical mechanics of hydration was applied to Msil RBD1-r(GUAG) interaction to provide a deeper understanding into the recognition mechanism [11]. Recently, we have also determined the NMR solution structure of Msil RBD2r(GUAGU) complex, elucidating the precise interactions between Msi1 RBD2 and $r(U A G)$ [12]. Comparison of the structures revealed that these RBDs utilize quite different interactions to recognize cognate RNA sequences. The minimal recognition sequences, which we identified, were actually found in many Msil-targeted mRNAs mentioned above $[10,12]$. On the basis of the two complex structures, we built a model structure of consecutive RBDs, RBD1-2, with r(UAGGUAG) containing both minimal recognition sequences; the result suggested that RBD1-2 should indeed be able to recognize r(UAGNnGUAG) (n $=0-50 \mathrm{nt})[12]$.
A considerable number of reports have shown that Msi1 is highly expressed in a broad range of human cancers and tumors [2,3], such as breast, colon, and lung cancers, astrocytomas, cervical carcinomas, ependymomas, endometrial carcinomas, gliomas, hepatomas, medulloblastomas, neurocytomas, and retinoblastomas. Msil is causally related to cancer initiation, progression, and drug resistance, however, a detailed description of signaling pathways that are regulated by Msil are still under investigation.

Recently, small-molecule inhibitors of Msi1 RBD1-RNA interaction were obtained. Lan et al. [13] screened $\sim 2000$ compound library against full-length Msil for inhibition of RNA-binding activity, and identified (-)-gossypol, which is a natural product extracted from cottonseed and had already completed phase IIb clinical trials for prostate cancer. Then, (-)-gossypol was shown to reduce Notch/Wnt signaling in colon cancer cell lines and suppress tumor growth in a mouse xenograft model. Furthermore, it was demonstrated that (-)-gossypol directly binds to the RNA-binding pocket of Msil RBD1. Likewise, Clingman et al. [14] performed high throughput screening and obtained 18-22 carbon $\omega-9$ monounsaturated fatty acids, which were then shown to inhibit proliferation of a cell line expressing Msil. The fatty acid was then shown to bind to Msi1 RBD1 and allosterically inhibit the RNAbinding. In both of the above studies $[13,14]$, NMR chemical shift data [BMRB ID: 11450] and NMR solution structure [PDB ID: 2RS2] of Msil RBD1 were used to identify the residues involved in compoundbinding and map the compound-binding sites on the structure.

These studies suggested that Msil is druggable and may have a potential to become a therapeutic target. Importantly, the development of compounds that exhibit a higher affinity and specificity toward Msil should provide a powerful tool to investigate and clarify the regulatory functions of Msi1 in normal and cancer cells. To develop better Msi1 inhibitors, it is important to understand the compound-binding mechanism of Msil. To this end, NMR chemical shift data and NMR solution structures of both Msi1 RBD1 and RBD2 in their free and RNA-complexed forms $[10,12,15]$ are expected to play important roles.

Correspondence to: Takashi Nagata, Institute of Advanced Energy, Graduate School of Energy Science, Kyoto University, Gokasho, Uji, Kyoto 611-0011, Japan, Tel: +81(774)38-3518 / 3519, Fax: +81(774)38-3524, E-mail: nagata. takashi.6w@kyoto-u.ac.jp

Received: March 11, 2018; Accepted: March 29, 2018; Published: April 02, 2018 


\section{References}

1. Sakakibara S, Imai T, Hamaguchi K, Okabe M, Aruga J, et al. (1996) Mouse-Musashi-1, a neural RNA-Binding protein highly enriched in the mammalian CNS stem cell. Dev Biol 176: 230-242. [Crossref]

2. Horisawa K, Yanagawa H (2012) In: Sun T (Edtr). Neural Stem Cells and Therapy. In Tech, Shanghai, 205-222.

3. Kudinov AE, Karanicolas J, Golemis EA, Boumber Y (2017) Musashi RNA-Binding Proteins as Cancer Drivers and Novel Therapeutic Targets. Clin Cancer Res 23: 2143 2153. [Crossref]

4. Spears E, Neufeld KL (2011) Novel double-negative feedback loop between adenomatous polyposis coli and Musashil in colon epithelia. J Biol Chem 286: 49464950. [Crossref]

5. Kawahara H, Imai $T$, Imataka $H$, Tsujimoto $M$, Matsumoto $K$, et al. (2008) Neural RNA-binding protein Musashi1 inhibits translation initiation by competing with eIF4G for PABP. J Cell Biol 181: 639-653. [Crossref]

6. Imai T, Tokunaga A, Yoshida T, Hashimoto M, Mikoshiba K, et al. (2001) The neural RNA-binding protein Musashil translationally regulates mammalian numb gene expression by interacting with its mRNA. Mol Cell Biol 21: 3888-3900. [Crossref]

7. Battelli C, Nikopoulos GN, Mitchell JG, Verdi JM (2006) The RNA-binding protein Musashi-1 regulates neural development through the translational repression of p21WAF-1. Mol Cell Neurosci 31: 85-96. [Crossref]

8. Miyanoiri Y, Kobayashi H, Imai T, Watanabe M, Nagata T, et al. (2003) Origin of higher affinity to RNA of the N-terminal RNA-binding domain than that of the C-terminal one of a mouse neural protein, Musashil, as revealed by comparison of their structures, modes of interaction, surface electrostatic potentials, and backbone dynamics. $J$ Biol Chem 278: 41309-41315. [Crossref]
9. Nagata T, Kanno R, Kurihara Y, Uesugi S, Imai T, et al. (1999) Structure, backbone dynamics and interactions with RNA of the C-terminal RNA-binding domain of a mouse neural RNA-binding protein, Musashi1. J Mol Biol 287: 315-330. [Crossref]

10. Ohyama T, Nagata T, Tsuda K, Kobayashi N, Imai T, et al. (2012) Structure of Musashi in a complex with target RNA: the role of aromatic stacking interactions. Nucleic Acids Res 40: 3218-3231. [Crossref]

11. Hayashi T, Matsuda T, Nagata T, Katahira M, Kinoshita M (2018) Mechanism of protein-RNA recognition: Analysis based on statistical mechanics of hydration. Phys Chem Chem Phys. 20: 9167-9180.

12. Iwaoka R, Nagata T, Tsuda K, Imai T, Okano H, et al. (2017) Structural Insight into the Recognition of $\mathrm{r}(\mathrm{UAG})$ by Musashi-1 RBD2, and Construction of a Model of Musashi-1 RBD1-2 Bound to the Minimum Target RNA. Molecules 22: e1207. [Crossref]

13. Lan L, Appelman C, Smith AR, Yu J, Larsen S, et al. (2015) Natural product (-)-gossypo inhibits colon cancer cell growth by targeting RNA-binding protein Musashi-1. Mol Oncol 9: 1406-1420. [Crossref]

14. Clingman CC, Deveau LM, Hay SA, Genga RM, Shandilya SM, et al. (2014) Allosteric inhibition of a stem cell RNA-binding protein by an intermediary metabolite. Elife 3: e02848. [Crossref]

15. Iwaoka R, Nagata T, Tsuda K, Imai T, Okano H, et al. (2017) Backbone and side chain assignments of the second RNA-binding domain of Musashi-1 in its free form and in complex with 5-mer RNA. Biomol NMR Assign 11: 265-268. [Crossref]

Copyright: ( 2018 Nagata T. This is an open-access article distributed under the terms of the Creative Commons Attribution License, which permits unrestricted use, distribution, and reproduction in any medium, provided the original author and source are credited. 